\title{
Studies of orbital parameters and pulse profile of the accreting millisecond pulsar XTE J1807-294
}

\author{
M. G. F. Kirsch ${ }^{1}$, K. Mukerjee ${ }^{4,5}$, M. G. Breitfellner ${ }^{1}$, S. Djavidnia ${ }^{1}$, M. J. Freyberg ${ }^{2}$, \\ E. Kendziorra ${ }^{3}$, and M. J. S. Smith ${ }^{1}$
}

\begin{abstract}
${ }^{1}$ European Space Agency (ESA), Research and Scientific Support Department (RSSD), Science Operations and Data System Division (SCI-SD), XMM-Newton Science Operations Centre, Apartado, PO Box 50727, 28080 Madrid, Spain e-mail: mkirsch@xmm.vilspa.esa.es

2 Max-Planck-Institut für extraterrestrische Physik, Giessenbachstrasse, 85748 Garching, Germany

3 Institut für Astronomie und Astrophysik Universität Tübingen, Abteilung Astronomie, Sand 1, 72076 Tübingen, Germany

4 Space Research Centre, Department of Physics and Astronomy, University of Leicester, Leicester, LE1 7RH, UK

5 Department of Astronomy and Astrophysics, Tata Institute of Fundamental Research, Colaba, Mumbai-400005, India
\end{abstract}

Received 2 February 2004 / Accepted 1 July 2004

\begin{abstract}
The accreting millisecond pulsar XTE J1807-294 was observed as a Target of Opportunity (ToO) by XMM-Newton on March 22, 2003 after its discovery on February 21, 2003 by RXTE. The source was detected in its bright phase with an observed average count rate of $33.3 \mathrm{cts} \mathrm{s}^{-1}$ in the EPIC-pn camera in the $0.5-10 \mathrm{keV}$ energy band (3.7 mCrab). Using the earlier established best-fit orbital period of $40.0741 \pm 0.0005 \mathrm{~min}$ from the RXTE observations and considering a circular binary orbit as first approximation, we derived a value of $4.8 \pm 0.1 \mathrm{lt}-\mathrm{ms}$ for the projected orbital radius of the binary system and an epoch of the orbital phase of MJD $52720.67415(16)$. The barycentric mean spin period of the pulsar was derived as $5.2459427 \pm 0.0000004 \mathrm{~ms}$. The pulsar's spin-pulse profile showed a prominent $(1.5 \mathrm{~ms} F W H M)$ pulse, with energy and orbital phase dependence in the amplitude and shape. The measured pulsed fraction in four energy bands was found to be $3.1 \pm 0.2 \%$ $(0.5-3.0 \mathrm{keV}), 5.4 \pm 0.4 \%(3.0-6.0 \mathrm{keV}), 5.1 \pm 0.7 \%(6.0-10.0 \mathrm{keV})$ and $3.7 \pm 0.2 \%(0.5-10.0 \mathrm{keV})$, respectively. Studies of spin-profiles with orbital phase and energy showed significant increase in its pulsed fraction during the second observed orbit of the neutron star, gradually declining in the subsequent two orbits, which was associated with sudden but marginal increase in mass accretion. From our investigations of orbital parameters and estimation of other properties of this compact binary system, we conclude that XTE J1807-294 is very likely a candidate for a millisecond radio pulsar.
\end{abstract}

Key words. stars: neutron star - pulsars: individual: XTE J1807-294 - accreting millisecond pulsar - XMM-Newton

\section{Introduction}

The accreting millisecond pulsar XTE J1807-294 was discovered as the fourth candidate of this class by RXTE (Markwardt et al. 2003a) on February 21, 2003. The coherent pulsation of $5.245902 \mathrm{~ms}$ was detected and subsequently the orbital period of $40.0741 \pm 0.0005$ min was established (Markward et al. 2003b), confirming it to be the shortest orbital period out of five such pulsar systems discovered so far. The spectral measurements of XTE J1807-294 showed a combination of absorbed black-body and a comptonization model with no absorption or emission lines (Campana et al. 2003). The other accreting millisecond pulsars known so far are SAX J1808.4-3658 (2.49 ms; Wijnands \& van der Klis 1998), XTE J1751-305 (2.30 ms; Markwardt \& Swank 2002), XTE J0929-314 (5.41 ms; Remillard et al. 2002), XTE J1807-294 (5.25 ms; Markwardt et al. 2003a) and the most recent XTE J1814-338 (3.18 ms; Strohmayer et al. 2003). Detailed studies on the first three candidates showed that these were short period,
X-ray transient, ultra compact binary systems having orbital periods $\leq 80$ min (Bildsten \& Chakrabarty 2001; Juett et al. 2003; Markwardt et al. 2002). The binary parameters imply $\approx 0.01-0.02 M_{\odot}$ white dwarf donors with moderately high inclination in these binaries (Galloway et al. 2002; Bildsten 2002; Markwardt et al. 2002).

Studies of the binary parameters of compact stars and their stellar companions provide a better understanding of the physical conditions of this new class of objects, their accretion physics and possible evolutionary connections to the class of radio pulsars (Bhattacharya \& van den Heuvel 1991). With this in mind, we have done a detailed analysis of the $\mathrm{ToO}$ data of XTE J1807-294 obtained by XMM-Newton on March 22, 2003. The spin-period of XTE J1807-294 has been confirmed, using XMM-Newton data and the projected semi-major axis of the binary orbit derived using a technique applicable to short period pulsars (Kirsch \& Kendziorra 2003). We present new results on XTE J1807-294 in this letter from our studies of orbital parameters, orbital phase and energy dependent pulse 
profiles, which provide better insight into the physical conditions of this pulsar system.

\section{XMM-Newton observations}

XTE J1807-294 was observed by XMM-Newton on March 22, 2003, starting at 2003-03-22 13:40:27 UT, with ObsId 01579601 in revolution number 601. The EPIC-pn CCD camera was operated in Timing mode with the Thick Filter. For this analysis, data from a 9293 s long exposure were used which covered almost four orbital periods of the source.

The EPIC-pn camera provides high time resolution in Timing mode $(0.03 \mathrm{~ms})$, and Burst mode $(7 \mu \mathrm{s})$ with moderate energy resolution $(E / \mathrm{d} E=10-50)$ in the $0.1-15 \mathrm{keV}$ energy band. This makes the pn-camera most suitable for simultaneous timing and spectral studies of millisecond pulsar sources.

\section{Data analysis}

\subsection{Data reduction}

The data were processed with SAS 5.4.1. Event times were corrected to the solar system barycentre with the $S A S$ tool barycen. In the Timing mode of the pn camera, a point source will be completely smeared out in the DETY direction. Hence, this mode provides only one-dimensional spatial information. Therefore, we used a nine column (37 arcsec) wide source extraction region centred on the source, consisting of CCD columns 33-41. CCD columns 03-11 were used for the background extraction region. An average counting rate of $33.7 \mathrm{cts} \mathrm{s}^{-1}$ was detected from the source in the $0.5-10 \mathrm{keV}$ band $(3.7 \mathrm{mCrab})$, while an average background of $0.4 \mathrm{cts} \mathrm{s}^{-1}$ was detected.

\section{2. $\chi^{2}$ grid search of the orbital parameters}

Using the best fit orbital period of $40.0741 \pm 0.0005 \mathrm{~min}$ (Markwardt et al. 2003b), and assuming a circular orbit, the relevant orbital parameters were determined through maximum $\chi^{2}$ epoch folding using 6 bin pulse profiles. To this end, the photon arrival times with respect to the binary barycentre of all events were corrected for different values of projected orbital radius $x_{0}$, and orbital phase $t_{0}$, where $x_{0}$ was varied in seven steps of 0.10 light-ms and $t_{0}$ in 14 steps equivalent to $1.0^{\circ}$, using $t_{\text {binary corrected }}=t-x_{0} \sin \left(2 \pi / P_{\text {orb }} \times\left(t-t_{0}\right)\right)$.

The initial parameter values were obtained by grouping events into 20 phase bins of the binary orbit and identifying two phases separated by $180^{\circ}$ via a $\chi^{2}$ epoch folding for each of the phase bins.

The results of the two-dimensional parameter space search are shown in Fig. 1. The data were interpolated through Delaunay triangulation in order to better to visualise the $\chi^{2} \mathrm{de}$ pendence on $x_{0}$ and $t_{0}$. A maximum $\chi^{2}$ of 520 was found for $x_{0}$ of $4.8 \pm 0.1$ light-ms and $t_{0}$ of $52720.67415(16)$ (MJD).

In order to test the assumption of a circular orbit, an analysis of possible orbital eccentricity of the neutron star orbit was carried out. However, no statistical significance for an eccentric orbit could be found and therefore the orbit is treated as

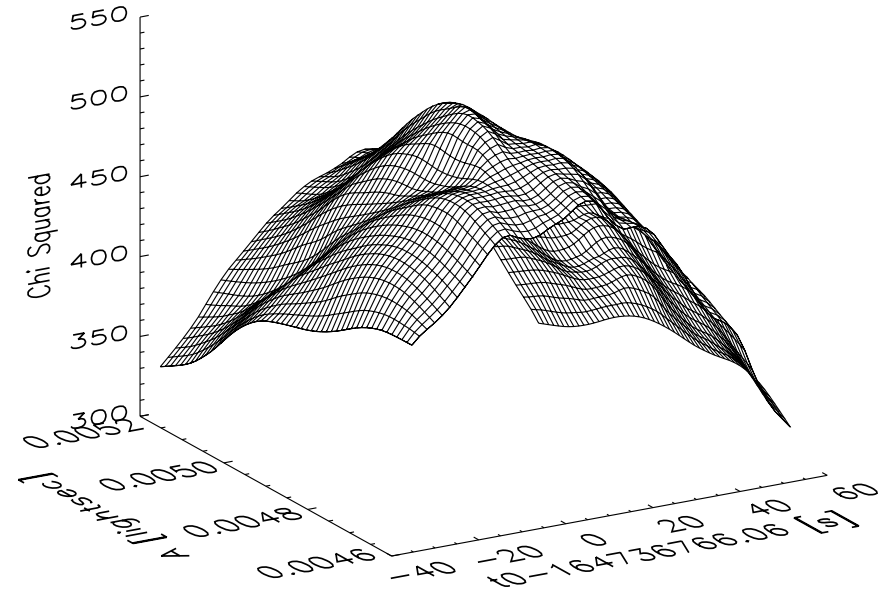

Fig. 1. $\chi^{2}$ grid search of the orbital parameters. See text for details.

Table 1. Measured parameters of XTE J1807-294.

\begin{tabular}{lcc}
\hline \hline Parameter & Value & Instrument \\
\hline RA & 18:07:00.0 (J2000) & Chandra $^{1}$ \\
Dec & $-29: 24: 30.0(\mathrm{~J} 2000)$ & Chandra $^{1}$ \\
Orbital period & $40.0741(5) \mathrm{min}$ & RXTE $^{1}$ \\
Spin period & $5.2459427(4) \mathrm{ms}$ & XMM-Newton $^{2}$ \\
Proj. orbital radius $x_{0}$ & $4.8(1)$ light-ms & XMM-Newton \\
Orbital phase $t_{0}$ & $52720.67415(16)(\mathrm{MJD})$ & XMM-Newton $^{2}$ \\
\hline
\end{tabular}

${ }^{1}$ Markward et al. (2003b); ${ }^{2}$ this work.

circular in all further analysis. Table 1 summarizes relevant parameters of the accreting millisecond pulsar XTE J1807-294 established so far.

\subsection{Pulse profile}

Using the best-fit orbital period of $40.0741 \pm 0.0005 \mathrm{~min}$ (Markwardt et al. 2003b) and the orbital parameters derived as described in Sect. 3.2, photon arrival times were corrected to the binary barycentre. We then derived the barycentric mean spin period of the pulsar of $5.2459427 \pm 0.0000004 \mathrm{~ms}$. By epoch folding the data with the derived period with respect to the epoch at MJD 52720.72457, we established spin pulse profiles in four different energy bands between $0.5-10 \mathrm{keV}$ as shown in Fig. 2. These profiles show a very prominent pulse $(1.5 \mathrm{~ms} F W H M)$. The shapes and relative strengths of the profile varies with energy. We determined the pulsed fraction in these energy bands using the expression

pulsed fraction $=\frac{\sum_{\text {bins }} \mid \text { flux per bin }- \text { mean flux per bin } \mid}{\text { total flux }}$

The measured pulsed fraction was found to be $3.1 \pm 0.2 \%$ $(0.5-3.0 \mathrm{keV}), 5.4 \pm 0.4 \%(3.0-6.0 \mathrm{keV}), 5.1 \pm 0.7 \%$ $(6.0-10.0 \mathrm{keV})$ and $3.7 \pm 0.2 \%(0.5-10.0 \mathrm{keV})$, respectively, in the four different energy bands as in Fig. 2.

To study the variation of the spin-pulse profile with orbital phase, we grouped the data in six different phases covering the complete binary orbit, where phase 0 starts at $t_{0}-44.08 \mathrm{~s}$. 


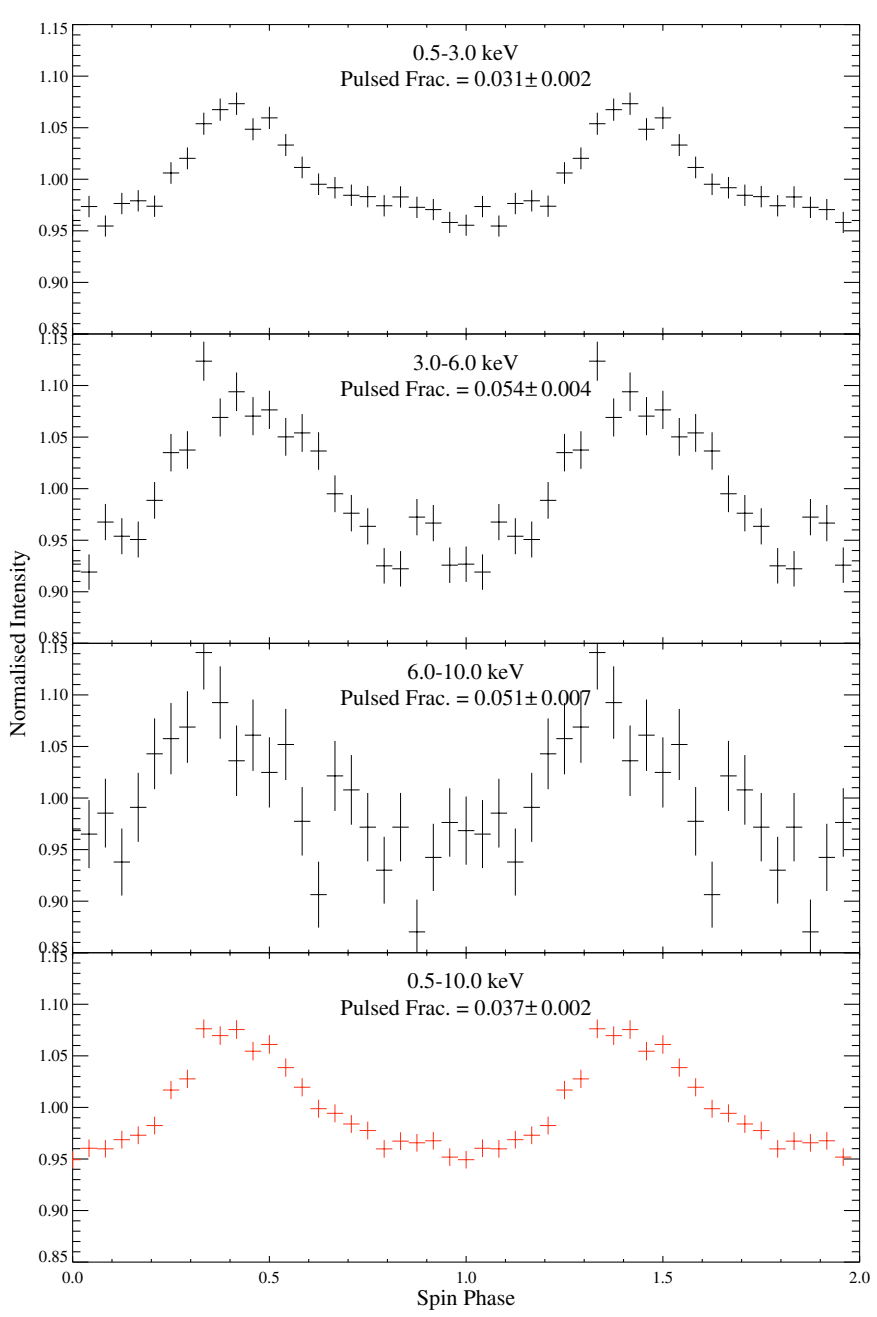

Fig. 2. Spin-pulse profiles of XTE J1807-289 in different energy bands.

The folded light curves corresponding to six orbital phases derived for the $0.5-10.0 \mathrm{keV}$ energy band are shown in Fig. 3 . The spin-pulse profile corresponding to orbital phase 3 , shows a significantly higher pulsed fraction. We then performed a detailed analysis for each of the four observed orbits and detected a significantly higher pulsed fraction corresponding to orbital phase 3 of orbit 2, which gradually declines during orbits 3 and 4 . Figure 4 gives the pulsed fraction calculated for six orbital phases for all the four binary orbits in the $0.5-3.0 \mathrm{keV}$ energy band.

\section{Discussion}

X-ray pulse profiles of XTE J1807-294 showed both energy and orbital phase dependence during the XMM-Newton observation. Pulse profiles of XTE J1807-294 show orbital phase dependent changes and variation of pulsed fraction as quoted in Fig. 3. These effects are also seen in other X-ray pulsars due to geometrical effects with respect to the line of sight and physical conditions of the pulsar (Wang \& Welter 1981). The sudden increase of the pulsed fraction value, as seen in Fig. 4, particularly during phase 3 in orbit 2, decreases gradually during subsequent orbits and is caused very likely by a sudden but

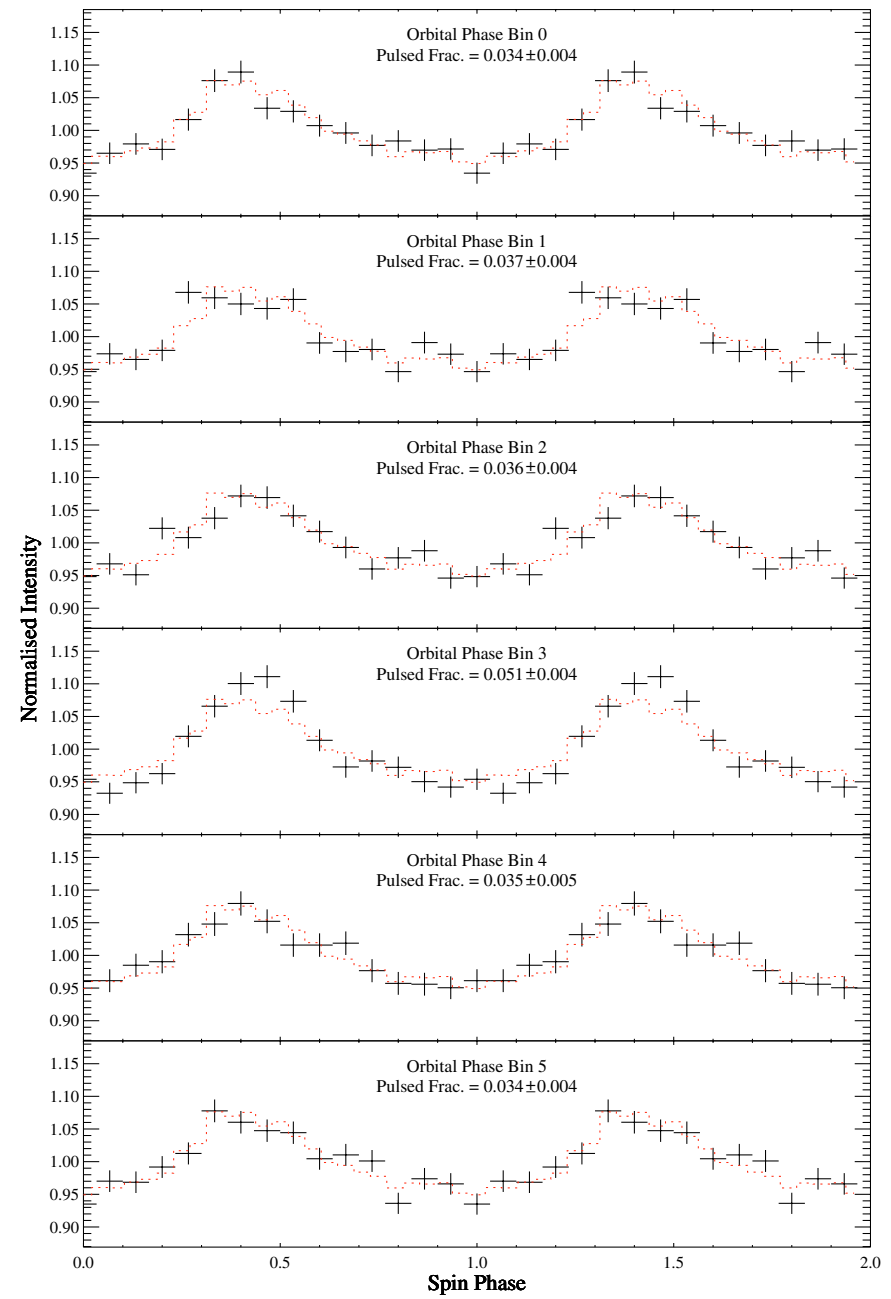

Fig. 3. Spin-pulse profiles of XTE J1807-289 at different orbit phases in the energy range $0.5-10 \mathrm{keV}$. In dotted red the orbit averaged folded spin light curve from Fig. 2 in the same energy range.

marginal increase in mass accretion. This causes an increase in the pulsed fraction of the pulsar which then gradually decreases due to the reduction of accreting matter and hence the pulsed fraction when neutron star poles are visible at the line of sight corresponding to a particular orbit phase 3 . Such changes in the pulse profiles and pulsed fraction due to increase in mass accretion rate during an outburst phase are commonly seen in X-ray binaries (Galloway et al. 2002; Mukerjee et al. 2000).

Determination of binary orbital parameters enables us to estimate properties of the accreting binary system. The short orbital period of 40.0741 min of XTE J1807-294 immediately confirms that it is the most compact binary system out of the five accreting millisecond pulsars known so far. Estimations of the semi-major axis $a \sin i$ for this binary using our technique described above give 0.0048 lt-s which enables us to estimate the mass function of this pulsar using the expression,

$f_{M}=1.074 \times 10^{-3} P^{2 / 3}(a \sin i / 1 \mathrm{lt}-\mathrm{s})^{3}\left(P_{\mathrm{orb}} / 1 \mathrm{~d}\right)^{-2} M_{\odot}$.

The pulsar mass function $f_{M}=1.6 \times 10^{-7} M_{\odot}$ is the smallest presently known for any stellar binary. For a neutron star mass of $1.4 M_{\odot}$, the minimum companion mass would be $0.007 M_{\odot}$ for an inclination angle of $i=90^{\circ}$, suggesting that the 


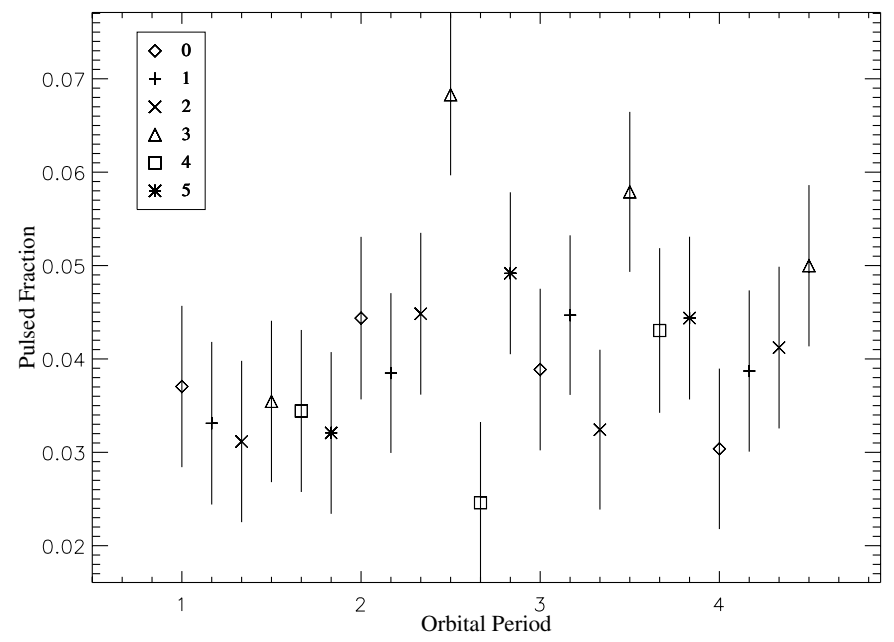

Fig. 4. Pulsed fraction values for 6 orbital phases indicated by different symbols for all the four orbits in the $0.5-3.0 \mathrm{keV}$ energy band.

companion of XTE J1807-294 is in the regime of very low mass dwarf, of the order of 7 Jupiter masses. The nature of the companion in an ultracompact binary system depends on prior evolution scenario. Binary systems with $P_{\text {orb }} \leq 80$ min can be formed in at least two possible evolution as summarized by Deloye \& Bildsten (2003) and the references therein. The first is, stable mass transfer on to a neutron star from an evolved main-sequence star or a He-burning star. In this scenario, the main sequence star, when its core has nearly completed hydrogen burning, is brought into Roche lobe contact due to loss of orbital angular momentum from magnetic braking which can evolve into a system with orbital periods comparable to those of accreting millisecond pulsars detected so far and can reach a minimum period of $10 \mathrm{~min}$ (Nelson et al. 1986; Podsiadlowski et al. 2002; Nelson \& Rappaport 2003) and when the system evolving through $40 \mathrm{~min}$ on the way out from the minimum period can have masses $\left(0.01 M_{\odot}\right)$ more in line with the measurements (Nelson \& Rappaport 2003). The second channel involves evolution through common-envelope phase during an unstable mass transfer from the white dwarf donor's progenitor star of either pure $\mathrm{He}$ or $\mathrm{C} / \mathrm{O}$ and are most recently discussed in detail by Deloye \& Bildsten (2003).

The composition of the donor in XTE J1807-294 could be $\mathrm{He} / \mathrm{H}$, pure $\mathrm{He}$ or a $\mathrm{C} / \mathrm{O}$ mixture depending on formation history. Without further data, the composition of the donor can not be determined. However, one can examine the nature of the donor based on orbital information of the binary system by applying models developed for ultra compact accreting millisecond pulsars by Deloye \& Bildsten (2003). Their model considers low-mass $\leq 0.1 M_{\odot}$ White dwarf companion of arbitrary degeneracy of evolved $\mathrm{He}$ or $\mathrm{C} / \mathrm{O}$ composition and central temperature of $10^{5}-10^{7}$ as range of relevance for these objects, the corresponding central densities $\left(10^{3} \mathrm{~g} \mathrm{~cm}^{-3}\right.$ are such that Coulomb and thermal contributions to the equation of state provide non-negligible corrections to degenerate electron pressure, affecting their $M-R$ relations. The model thus based on equation of state, adequately describe relevant physics and yields analytic description of the qualitative behavior of $M-R$ relations and how they are affected by Coulomb and thermal contributions to the equation of state. The model established a relationship between the orbital inclination for ultra compact binaries and the donor's composition and central core temperature. The application of this model to three of the known accreting millisecond pulsar systems suggest that if the donors in all the three systems are He White dwarfs, the $T=0$ objects are allowed in XTE J0929-314 and XTE J1807-294 while XTE J1751-305 requires a hot donor (Bildsten 2002). While $\mathrm{C} / \mathrm{O}$ cold donor is possible for XTE J1807-294, the other two systems required hot C/O donors. For XTE J1807-294 in particular, with measured orbital parameters suggest that the companion for this ultra compact binary system has greater phase space available for a C/O donor than a He donor. Because for XTE J1807-294, the apriori probability greatly favors $\mathrm{C} / \mathrm{O}$ donor in terms of allowed inclination as the probability that a donor is $\mathrm{He}$ is only $15 \%$ in this system (Deloye \& Bildsten 2003). Further evolution of this system will depend on its orbital inclination which in terms depends on donor mass, core temperature and its composition. From known properties of this binary system, it is likely that at the end of its accretion phase, it could be a candidate for a millisecond radio pulsar.

Acknowledgements. The XMM-Newton project is an ESA Science Mission with instruments and contributions directly funded by ESA Member States and the USA (NASA). The German contribution of the XMM-Newton project is supported by the Bundesministerium für Bildung und Forschung/Deutsches Zentrum für Luft- und Raumfahrt. We thank an anonymous referee for his very constructive comments.

\section{References}

Bhattacharya, D., \& van den Heuvel, E. P. J. 1991, Phys. Rep., 203, 1 Bildsten, L. 2002, ApJ, 577, L27

Bildsten, L., \& Chakrabarty, D. 2001, ApJ, 557, 292

Campana, S., Ravasio, M., Israel, G. L., Mangano, V., \& Belloni, T. 2003, ApJ, 594, L39

Deloye, C. J., \& Bildsten, L. 2003, ApJ, 598, 1217

Galloway, D. K., Chakrabarty, D., Morgan, E. H., \& Remmilard, R. A. 2002, ApJ, 576, L137

Juett, A. M., Galloway, D. K., \& Chakrabarty, D. 2003, ApJ, 587, 754

Kirsch, M. G. F., \& Kendziorra, E. 2003, ATEL, 148

Markwardt, C. B., \& Swank, J. H. 2002, IAU Circ., 7867

Markwardt, C. B., Swank, J. H., Strohmayer, T. E., In't Zand, J. J. M., \& Marshall, F. E. 2002, ApJ, 575, L21

Markwardt, C. B., Smith, E., \& Swank, J. H. 2003a, IAU Circ., 8080 Markwardt, C. B., Juda, M., \& Swank, J. H. 2003b, IAU Circ., 8095

Mukerjee, K., Agrawal, P. C., Paul, B., et al. 2000, A\&A, 353, 239

Nelson, L. A., Rappaport, S. A., \& Joss, P. C. 1986, ApJ, 304, 231

Nelson, L. A., \& Rappaport, S. 2003, ApJ, 598, 431

Podsiadlowski, Ph., Rappaport, S., \& Pfahl, E. D. 2002, ApJ, 565, 1107

Strohmayer, T. E., Markwardt, C. B., Swank, J. H., et al. 2003, ApJ, 596, L67

Remillard, R. A., Swank, J., \& Strohmayer, T. 2002, IAU Circ., 7893

Wang, Y. M., \& Welter, G. L. 1981, A\&A, 102, 97

Wijnands, R., \& van der Klis, M. 1998, Nature, 394, 344 